\title{
Prevalence of SOS-mediated control of integron integrase expression as an adaptive trait of chromosomal and mobile integrons
}

\author{
Guillaume Cambray ${ }^{1 \dagger}$, Neus Sanchez-Alberola ${ }^{2,3 \dagger}$, Susana Campoy ${ }^{2}$, Émilie Guerin ${ }^{4}$, Sandra Da Re ${ }^{4}$, \\ Bruno González-Zorn ${ }^{5}$, Marie-Cécile Ploy ${ }^{4}$, Jordi Barbé ${ }^{3}$, Didier Mazel ${ }^{1^{*}}$ and Ivan Erill ${ }^{3^{*}}$
}

\begin{abstract}
Background: Integrons are found in hundreds of environmental bacterial species, but are mainly known as the agents responsible for the capture and spread of antibiotic-resistance determinants between Gram-negative pathogens. The SOS response is a regulatory network under control of the repressor protein LexA targeted at addressing DNA damage, thus promoting genetic variation in times of stress. We recently reported a direct link between the SOS response and the expression of integron integrases in Vibrio cholerae and a plasmid-borne class 1 mobile integron. SOS regulation enhances cassette swapping and capture in stressful conditions, while freezing the integron in steady environments. We conducted a systematic study of available integron integrase promoter sequences to analyze the extent of this relationship across the Bacteria domain.

Results: Our results showed that LexA controls the expression of a large fraction of integron integrases by binding to Escherichia coli-like LexA binding sites. In addition, the results provide experimental validation of LexA control of the integrase gene for another Vibrio chromosomal integron and for a multiresistance plasmid harboring two integrons. There was a significant correlation between lack of LexA control and predicted inactivation of integrase genes, even though experimental evidence also indicates that LexA regulation may be lost to enhance expression of integron cassettes.

Conclusions: Ancestral-state reconstruction on an integron integrase phylogeny led us to conclude that the ancestral integron was already regulated by LexA. The data also indicated that SOS regulation has been actively preserved in mobile integrons and large chromosomal integrons, suggesting that unregulated integrase activity is selected against. Nonetheless, additional adaptations have probably arisen to cope with unregulated integrase activity. Identifying them may be fundamental in deciphering the uneven distribution of integrons in the Bacteria domain.
\end{abstract}

\section{Background}

Integrons are bacterial genetic elements capable of incorporating exogenous and promoterless open reading frames (ORF), referred to as gene cassettes, by site-specific recombination (Figure 1). First described in the late 1980s in connection with the emergence of antibiotic resistance [1], integrons always contain three functional components: an

\footnotetext{
* Correspondence: mazel@pasteur.fr; erill@umbc.edu

† Contributed equally

'Institut Pasteur, Unité Plasticité du Génome Bactérien, CNRS URA 2171،

75015 Paris, France

${ }^{3}$ Department of Biological Sciences, University of Maryland Baltimore County,

Baltimore 21228, USA

Full list of author information is available at the end of the article
}

integrase gene (intI), which mediates recombination; a primary recombination site (attl); and an outward-orientated promoter $\left(P_{C}\right)$ [2]. Cassette integrations occur mainly at the attI site, ensuring the correct expression of newly captured cassettes by placing them under the control of the $P_{C}$ promoter [3,4]. To date, two main subsets of integrons have been described. On the one hand, mobile integrons, also referred to as multiresistance integrons, contain relatively few (two to eight) cassettes, and collectively encode resistance to a broad spectrum of antibiotics [5-7]. They have been conventionally divided into five different classes according to their intI gene sequence: class 1 for intI1, class 2 for intI2, class 3 for intI3, class 4 for intISXT (formerly intI9) and class 5 for intIHS [8,9]. Mobile integrons

\section{Biomed Central}




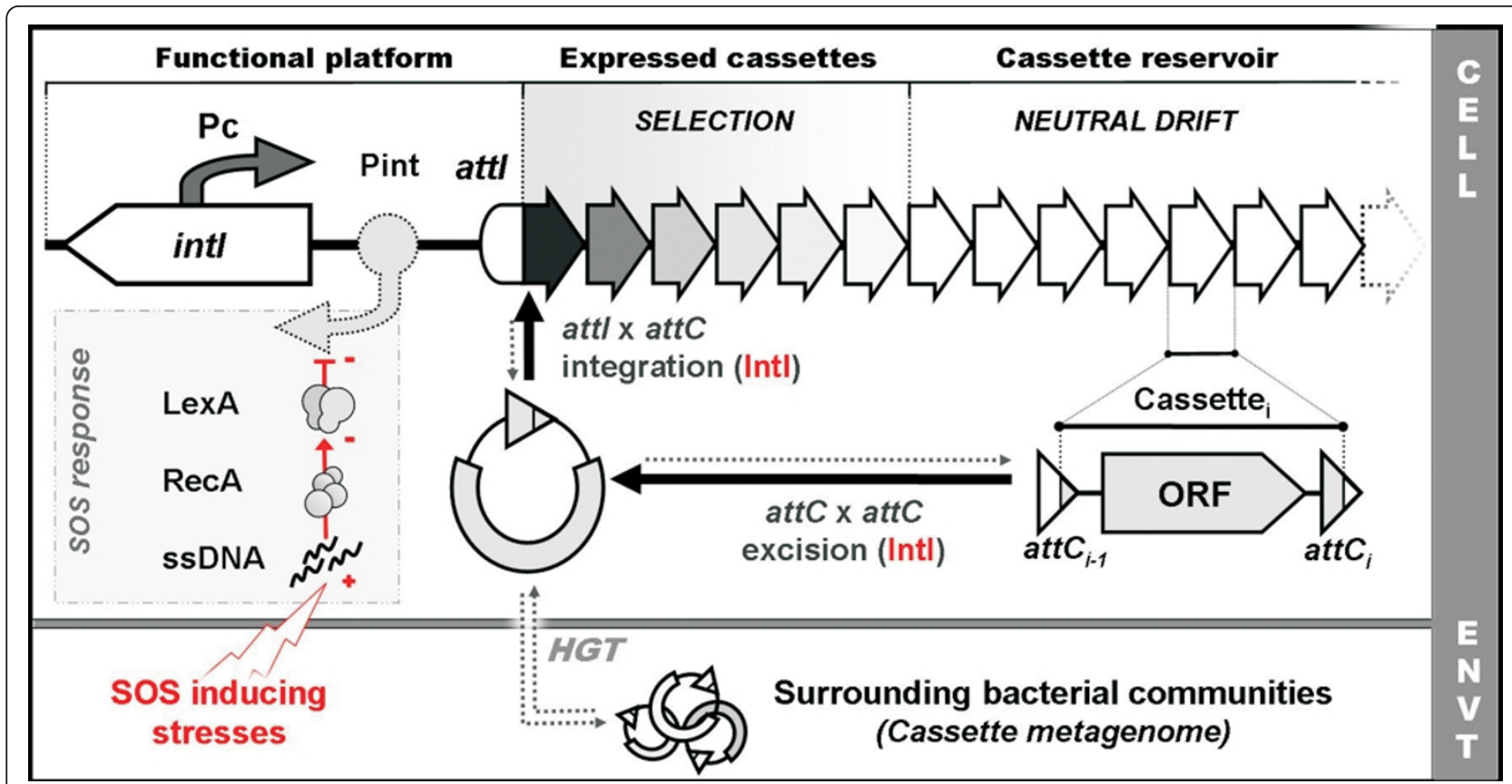

Figure 1 Schematic organization of integrons. The functional platform of integrons is constituted by an int/ gene encoding an integrase, its own promoter $P_{\text {int }}$ a cassette promoter $P_{C_{1}}$ and a primary recombination site attl. The system maintains an array that can consist of more than 200 cassettes. Only the few first cassettes are strongly expressed by the $P_{C}$ promoter, as indicated by the fading fill color. Cassettes generally contain a promoterless open reading frame (ORF) flanked by two recombination sites termed attC. Cassettes can be excised from any position in the array through attC $\times$ attC recombination mediated by the integrase. The resulting circular intermediate can then be integrated by the integrase, preferentially at attl. Exogenous circular intermediates can also be integrated, owing to the low specificity of the integrase activity, rendering the system prone to horizontal transfer. The SOS response directly controls the expression of many integron integrases by binding of its repressor protein, LexA, to a target site in the $P_{\text {int }}$ promoter.

are typically associated with transposons and conjugative plasmids, ensuring their dissemination across bacterial species. They are present mostly in the Proteobacteria, but have also been reported in other bacterial phyla, such as Gram-positive bacteria [9]. By contrast, chromosomal integrons have been identified in the genomes of many bacterial species [10]. Because their phylogeny reflects a predominant pattern of vertical inheritance, these integrons are not catalogued based on the class nomenclature described above, but according to their host species $[8,9]$. A subfamily of these, termed superintegrons (SIs), has been specifically identified in the Vibrionaceae and, to some extent, in the Xanthomonadaceae and Pseudomonadaceae [11-16]. Superintegrons typically encompass between 20 and 200 cassettes with species-specific sequence signatures [9], and seem to be ancient residents of the host genome [13]. Most of the genes in the superintegron cassettes are of unknown function [10], but some of them are related to existing resistance cassettes [17-20]. Although stable under laboratory conditions, superintegrons have been reported to be the most variable loci of $V$. cholerae natural isolates [12,21], suggesting that integron reorganization might be occasionally upregulated in natural environments. Integron integrases mediate recombination by interacting with single-stranded (ss) attC sites present in all reported cassettes, employing a unique, site-specific recombination process [22-24]. Despite the importance of integrons in the acquisition and spread of antibiotic-resistance determinants and, from a broader perspective, in bacterial adaptation, little was known about the regulatory control and dynamics of cassette recombination until recently, when we reported that the expression of the integron integrases in the $V$. cholerae superintegron and in a class 1 mobile integron was controlled by the SOS response [25].

The SOS response is a global regulatory network governed by a repressor protein (LexA) and principally targeted at addressing DNA damage [26,27]. LexA represses SOS genes by binding to highly specific binding sites present in their promoter regions. In $E$. coli and most $\beta$ - and $\gamma$-Proteobacteria, these sites consist of a palindromic motif (CTGTatatatatACAG) 16 bp long, commonly known as the LexA box [26]. The SOS response is typically induced by the presence of ssDNA fragments, which can arise from a number of environmental stresses [28], but are typically linked to replication-fork stall caused by DNA lesions. These ssDNA fragments bind non-specifically to the universal recombination protein 
RecA [29], enabling it to promote LexA inactivation by autocatalytic cleavage [30], and thus inducing the SOS response. Up to 40 genes have been shown to be directly regulated by LexA in E. coli [31], encoding proteins that stabilize the replication fork, repair DNA, promote translesion synthesis and arrest cell division. Since its initial description in E. coli [26], the SOS response has been characterized in many other bacterial classes and phyla, and LexA has been shown to recognize markedly divergent motifs in different bacterial groups [27].

In recent years, the SOS response has been linked to clinically relevant phenotypes, such as the activation and dissemination of virulence factors carried in bacteriophages [32-34], transposons [35] pathogenicity islands [36] and in integrating conjugative elements encoding antibiotic-resistance genes $[27,37,38]$. Moreover, it has recently become established that some widely used antibiotics, such as fluoroquinolones, trimethoprim and $\beta$-lactams, are able to trigger SOS induction and are thus able to promote the dissemination of antibiotic-resistance genes $[27,37,39-42]$. This puts forward a positive feedback loop that has been suggested to have important consequences for the emergence and dissemination of antibiotic resistance [43]. Our recent work, showing a link between the SOS response and integrase-mediated recombination [25] further reinforces this line of reasoning. Such a link provides bacteria with an antibioticinduced mechanism for gene acquisition, reorganization and dispersal. In hindsight, the coupling of genetic elements capable of cassette integration with a global response to stress comes out as an elegant and powerful pairing. Integrons can be seen as stockpiling agents of genetic diversity that, in addition, can tap into a huge and variable pool of cassettes through horizontal gene transfer from the surrounding bacterial communities (Figure 1) [10,44]. Nonetheless, efficient expression of these acquired traits is strongly dependent on integrasemediated recombination. Newly acquired cassettes sitting in the proximal region of the integron are strongly expressed by the $P_{C}$ promoter, but they can be displaced gradually to distal parts of the integron by the incorporation of new cassettes, and can thus become partially silenced. Infrequent excision and integration events can also relocate cassettes, moving them to distal or proximal parts of the integron, and thus have the ability to reinstate previously acquired cassettes (Figure 1). The timing of all these events is therefore of fundamental importance, and depends on the regulatory systems controlling the expression of the integron integrase gene. In this context, the discovery of a link between the SOS response and integrase expression is an important first step towards unraveling the precise mechanisms controlling integrase expression.
In this study, we expanded on this recent connection between the SOS response and integron integrase expression by means of a systematic study of integron integrase promoter regions. By combining in silico and in vitro methods, we show that LexA control of integrase expression is a widespread phenomenon that arose very early in the evolutionary history of integrons and has since been maintained through positive selection in mobile integrons and large chromosomal integrons. We report a significant correlation between the loss of LexA control and integrase inactivation, indicating that unregulated recombination may be deleterious in these genetic elements. Exceptions to this rule suggest that secondary adaptations to tolerate unregulated integrase expression may have arisen in some clades, and that the identification of such adaptations might shed light onto the uneven distribution of integrons in the Bacteria domain. We discuss these findings for the adaptive dynamics of integrons, and their implications for the acquisition and dissemination of antibiotic-resistance determinants.

\section{Results and discussion}

\section{Identification of LexA binding sites in intl promoters}

We recently identified E. coli-like LexA binding sites in the promoter region of intI1 integrase genes from mobile integrons and of the intIA integrase from the $V$. cholerae superintegron (Figure 2AB). In $V$. cholerae and some of these mobile integrons, the identified LexA boxes partially overlap the -10 element of the intI promoter in a classic operator organization. We have shown that expression of $V$. cholerae and E. coli pAT674 integrase genes is indeed controlled by the SOS response, leading to heightened rates of integrase-mediated recombination upon SOS induction [25].

To gain insight into the general relevance of this observation, we undertook an exhaustive in silico study of integrase regulation by the LexA protein. Using a TBLASTN search (National Center for Biotechnology Information (NCBI); http://blast.ncbi.nlm.nih.gov/), we identified 1,483 homologs of intIA in the non-redundant (NR) (971), environmental (ENV) (381) and Whole Genome Shotgun (WGS) (131) subdivisions of the GenBank database. When sufficient data were available (1,103 sequences), the nucleotide sequences corresponding to the first $50 \mathrm{bp}$ of the coding region plus $100 \mathrm{bp}$ upstream of the translation start site $(-100,+50)$ were systematically searched for LexA binding sites. We conducted independent searches for all the 15 LexA binding motifs described to date in the literature [27]. Putative LexA binding sites were detected in $56.6 \%$ (624) of the 1,103 sequences for which the $(-100,+$ 50) region was available (see Additional file 1), with 40 sequences displaying two LexA binding sites in tandem. All the identified LexA binding sites corresponded exclusively 


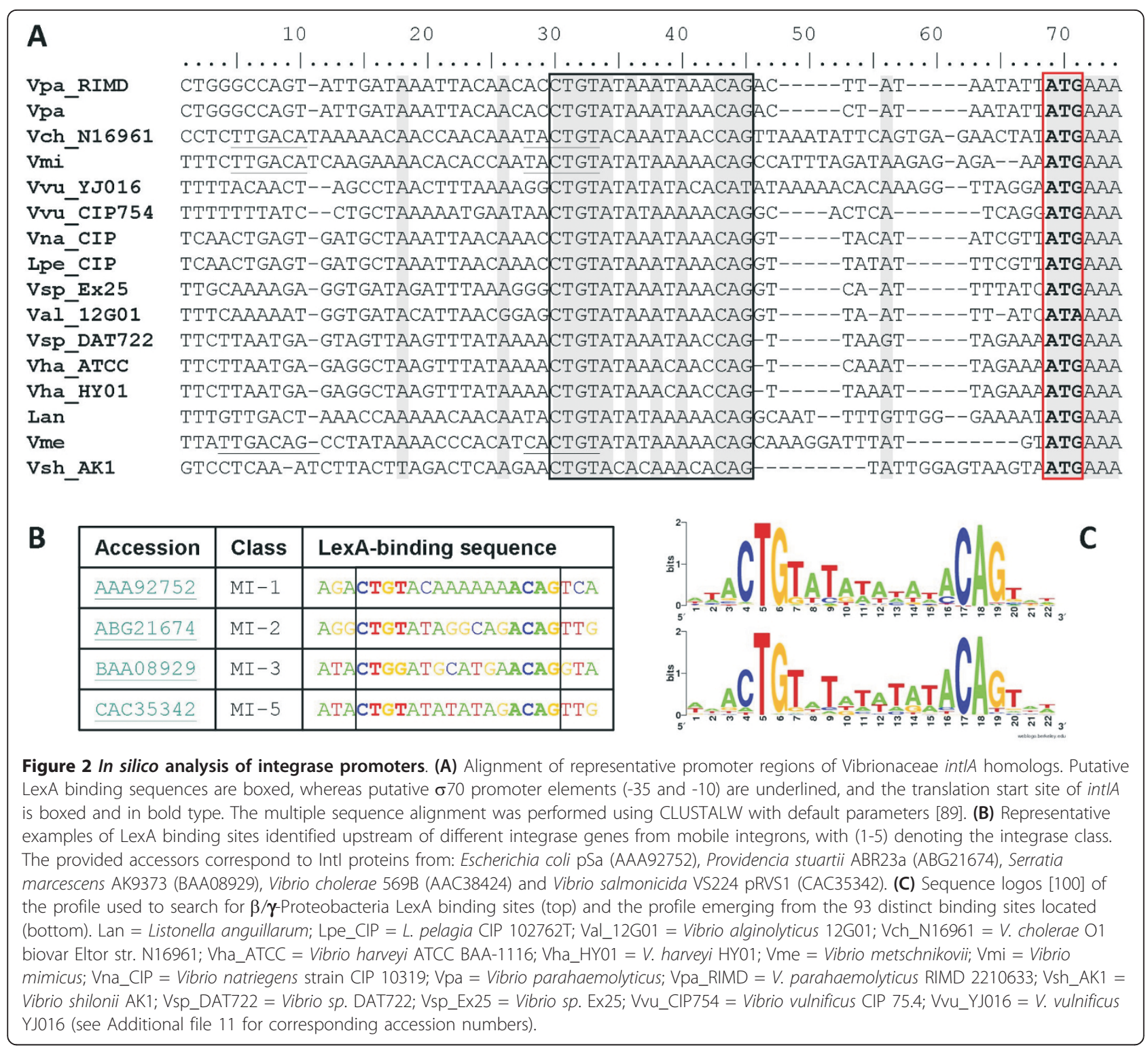

to the motif found in E. coli and most $\beta / \gamma$-Proteobacteria (Figure 2C). Given that we searched for 14 additional LexA binding motifs and that the sample of integrase sequences contained representatives from the respective clades in which these motifs have been reported, including one $\alpha$ Proteobacteria species, this strongly suggests that the putative LexA regulation of intI genes is restricted to organisms harboring LexA proteins that are able to recognize the $\beta / \gamma$ Proteobacteria. The LexA binding motif of the $\beta / \gamma$-Proteobacteria is markedly divergent from that seen in E. coli and the $\beta / \gamma$-Proteobacteria, and it is known to have arisen after the split of the $\alpha$ - and $\beta / \gamma$-Proteobacteria subclasses [45-49]. Hence, it seems very likely that LexA regulation of integrase genes also arose after this evolutionary branching point. When we examined the core $16 \mathrm{bp}$ sequence of the identified E. coli-like LexA binding sites, we identified 93 distinct sequences (see Additional file 2). These LexA binding sites presented substantial diversity while maintaining a high level of conservation, as reflected in their joint information content logo (Figure 2C). Importantly, E. coli-like LexA sites were detected in almost all Vibrionaceae superintegrons (Figure 2A), and in all but one of the mobile integron classes (Figure 2B), indicating that putative LexA regulation of intI genes is a widespread phenomenon among integrons.

\section{Predicted LexA binding sites correspond to functional transcriptional-control elements}

We have previously shown that LexA regulates the expression of intI in V. cholerae, and our in silico search 
identified LexA binding sites in the promoter region of intI for all sequenced Vibrio species (see Additional file 1 ). To further assess the overall functionality of the in silico predicted LexA binding sites, we evaluated integrase LexA regulation in Vibrio parahaemolyticus strain ATCC 17802, which harbors a LexA binding site upstream of its intIA gene in a genomic context that is substantially different from that of $V$. cholerae (Figure 2A). Using quantitative reverse transcriptase (RT)-PCR, we determined the intIA expression level in both the wild-type strain and its lexA(Def) derivative (lacking a functional $\operatorname{lex} A$ gene). We found an expression ratio of 9.28, revealing a strong LexA regulation of the intIA gene expression (Figure 3A). Furthermore, electrophoretic mobility-shift assays (EMSA) with purified $V$. parahaemolyticus LexA protein showed that the observed upregulation of intIA expression was directly mediated by LexA in this organism (Figure 3A).

In several class 1 integrons, heightened expression of the cassette genes has been shown to rely on a secondary cassette promoter called $P_{C 2}$, located just upstream of the intI1 gene (see Additional file 3 ). $P_{C 2}$ is enabled by a GGG insertion (on the top strand) that increases the distance between the -35 box sequence and a sequence resembling the -10 box consensus from 14 to $17 \mathrm{bp}$, thereby generating a functional $\sigma 70$ promoter $[3,4,50,51]$. In all its reported instances, this GGG insertion disrupts a seemingly functional LexA binding site. Therefore, it is likely that the GGG insertion that enables $P_{C 2}$ should simultaneously abolish integrase regulation by LexA. We tested this hypothesis using the E. coli multi-resistant plasmid pMUR050 [52], which provides an ideal background to test this hypothesis because it harbors two integrons with inactivated copies of the intI1 gene. The promoter regions of both intI genes are almost identical, and differ only in that one $\left(P_{\text {intII }}{ }^{-}\right)$contains a functional LexA binding site in its promoter, whereas the other $\left(P_{\text {intII }}{ }^{+}\right)$presents the aforementioned GGG insertion, disrupting the LexA binding site and enabling the $P_{C 2}$ promoter (see Additional file 3). As expected, EMSA confirmed that $E$. coli LexA is able to bind the $P_{\text {intII }}{ }^{-}$promoter, but that the GGG insertion effectively prevents LexA binding on $P_{\text {intII }}{ }^{+}$(Figure 3B). Furthermore, RTPCR in wild-type and lexA-defective backgrounds confirmed that LexA regulation was only present in the IntI $11^{-}$integrase carrying the intact LexA binding site, with a strong deregulation (ratio of 6.55) in the lexA mutant (Figure 3B). Thus, the GGG insert not only enables the secondary cassette promoter $P_{C 2}$, but concomitantly disrupts the LexA binding site of the integrase promoter.

To check whether the GGG insert did in fact lead to the activation of $P_{C 2}$ and increased cassette expression in the pMUR IntI1 ${ }^{+}$integrase, we compared RT-PCR expression profiles for the first cassette gene of both
pMUR integrons. We found that cassette-gene expression was enhanced in the integron containing the GGG insertion, and that this increase was independent of LexA (data not shown). In silico searches for disrupted LexA binding sites found 44 instances of similar GGG inserts in integrons from a wide variety of species (see Additional file 4), all corresponding to class 1 mobile integrons harboring multiple antibiotic-resistance cassettes. Together, these results suggest that LexA regulation may eventually be lost under heavy selection to promote higher basal levels of the antibiotic-resistance transcript.

\section{Ancestral-state reconstruction of LexA regulation and integrase functionality}

The presence of confirmed LexA regulation in $V$. cholerae and $V$. parahaemolyticus superintegrons suggested that SOS control of intI genes probably originated very early in their evolutionary history. Likewise, the complete absence of LexA binding motifs different from that of $E$. coli in all the intI promoters analyzed in this study indicated that LexA regulation must have been lost in integrons borne by species without LexA, or in which LexA recognizes a divergent motif $[27,47]$. At the same time, there is ample evidence of extensive (10\% to $30 \%$ ) and independent integrase inactivation across the Bacteria domain, implying that loss of integrase functionality may be an adaptive trait under particular selective pressures [53]. In this respect, the evidence of integrase inactivation in bacterial groups in which it is known that LexA does not recognize the E. coli motif $[16,54,55]$, such as the Xanthomonadales, suggests that loss of LexA regulation might be linked to mutational inactivation of the integrase gene.

To explore this hypothesis, we developed an automated system to assess integrase functionality based on the detection of generic (nonsense and indels) and integrasespecific missense mutations known to inactivate the protein (see Methods). This method was applied to 1,135 intIA homologs identified in this work for which sufficient coding sequence was available. Consistent with previous results, we found that a substantial fraction of integrase genes (43\%, see Additional file 5) seem to be inactivated [53]. For the 755 intIA homologs with sufficient sequence to apply both analyses, the predicted inactivation status for each integrase sequence (active/inactive) was combined with the predicted presence of a LexA binding site in its promoter $(-100,+50)$ region as computed previously. A correlation analysis was carried out to determine the existence of a link between loss of LexA regulation and integrase inactivation. The results of this analysis showed a significant correlation (Pearson $r=0.58$, Spearman $\rho=$ $0.53 ; \mathrm{P}<0.001$ ) between both traits (Figure 4), and give credence to the idea that loss of LexA regulation is associated with integrase inactivation. 


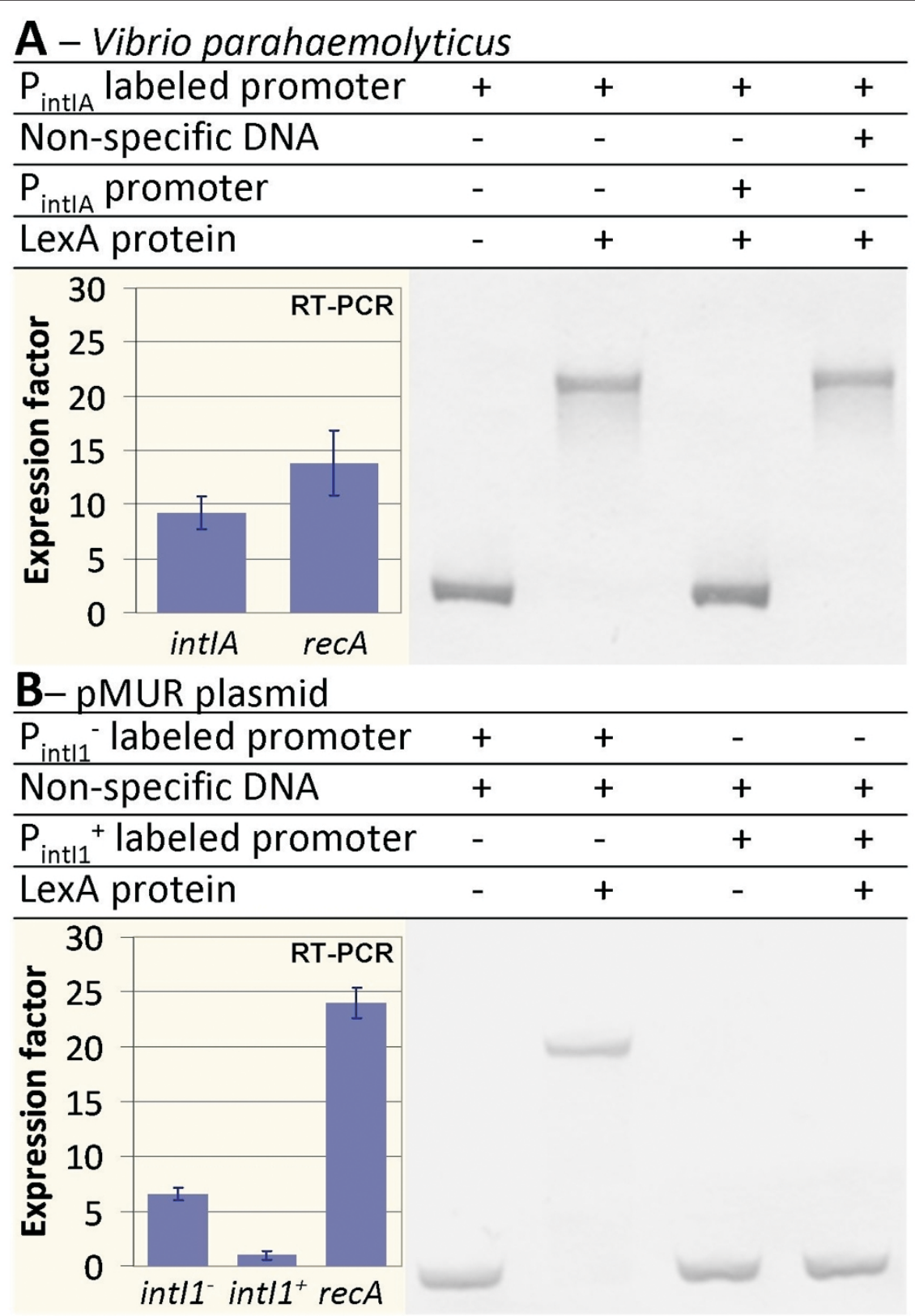

Figure 3 Electrophoretic mobility-shift assay (EMSA) and quantitative real-time reverse transcription PCR on different intl genes and their respective promoters. (A) Vibrio parahaemolyticus integron. EMSA of $V$. parahaemolyticus intIA promoter with purified $V$. parahaemolyticus LexA protein. Competitive assays using either non-specific or $P_{\text {int }}$ non-labeled DNA are also shown. The intA expression factor was calculated as the ratio of the relative intA mRNA concentration in the $V$. parahaemolyticus lexA mutant strain with respect to that in the wild type. (B) $E$. coli pMUR050 integrons. EMSA of pMUR050 int17 and int1/ (containing GGG insertion) promoters with purified E. coli LexA protein. The expression factor for both intl genes was calculated as the ratio of each relative intl mRNA concentration in the E. coli lexA-sulA mutant strain with respect to that in the wild type. In all cases, the expression factor of recA is shown as a control, and all expression factors are the mean value from three independent experiments (each in triplicate).

To gain insight into the evolutionary history of this correlation, we generated a phylogenetic tree of 44 representative IntI sequences, and applied ancestral-state reconstruction methods for both phenotypic characters (predicted integrase functionality and LexA regulation).
The tree (Figure 5) is in overall agreement with previously published IntI phylogenies $[9,53,56]$. As in previous phylogenies, two major ecological groups can be outlined on the tree: marine and freshwater/soil bacteria. Chromosomal superintegrons and class 5 mobile 


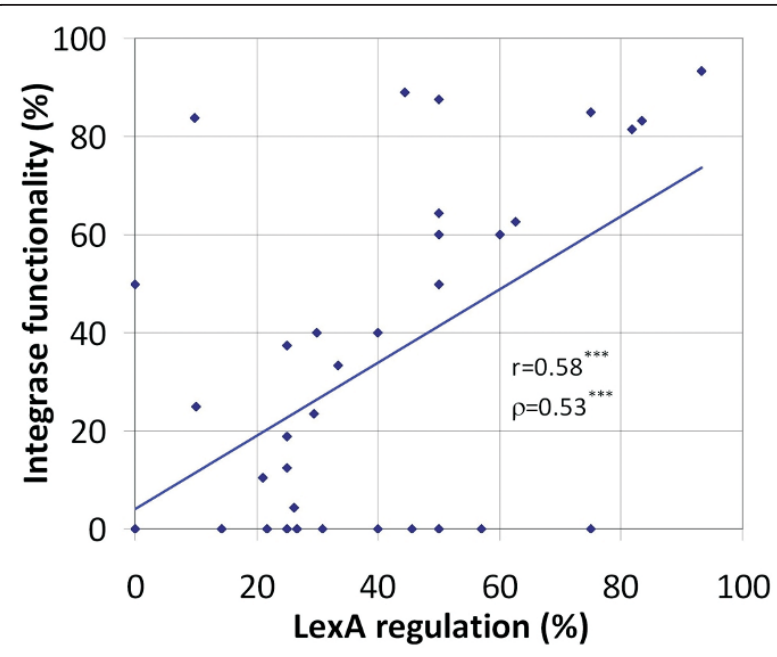

Figure 4 Correlation between inferred LexA regulation and integron integrase functionality. The plot was generated from the frequency values for each trait at each reference panel taxon, as derived from reciprocal BLAST mapping (see Additional file 14). Pearson and Spearman rank correlations and their respective $P$ values were computed in Excel (Microsoft Corp., Redmond, WA, USA). The asterisk rating system is used for correlation $P$ values (***P $<0.001)$. $P$ values are relative to two-tailed Student $t$-test on the null hypothesis (no correlation).

integrons borne by marine species form a monophyletic clade that sits at the root of the tree. From this early branch, a second radiation of integrons encompassing both chromosomal integrons and all other mobile integron classes splits neatly into integrons borne by, respectively, marine and soil/freshwater bacteria. In the marine species, class 2 and 4 mobile integrons form a monophyletic cluster with Shewanella chromosomal integrons that is also in agreement with previous analyses $[57,58]$. In the soil/freshwater clade, class 1 and 3 mobile integrases form a distinct group, suggesting an early split from their chromosomal counterparts in the Proteobacteria [59].

Both parsimony and maximum likelihood (ML) reconstructions of the ancestral state for LexA regulation strongly supported the notion that this feature was present in the common ancestor of bacterial integrons. LexA regulation (Figure 5, filled circles) is pervasive among Vibrio superintegrons and is also widespread within the marine integron radiation. It is also most likely (0.7 likelihood in ML reconstruction, see Additional file 6) that LexA regulation was present in the ancestor of the soil/freshwater radiation, and has been subsequently lost (Figure 5, open circles) in many of its internal clades. A notable exception to this trend are the class 1 and class 3 integrons, in which LexA regulation is still the norm. Our results thus imply that some particular trait in the environment of both chromosomal superintegrons and mobile integrons must be exerting a considerable selective pressure towards preservation of integrase LexA regulation. In the chromosomal integrons of the Vibrionaceae, the most likely source of this pressure is the stabilization of large integrons, which may include essential genes [15]. In mobile integrons, it seems likely that selection might favor integrons that remain largely inactive, but are capable of generating sharp bursts of recombination activity in times of need for evolutionary innovation.

The reconstruction of ancestral states for inferred integrase functionality is relatively congruent with the hypothesis that the loss of LexA regulation might be associated with integrase inactivation (Figure 5; see Additional file 7). Even though there is testimonial evidence of inactivation (Figure 5, dotted lines), integrases from almost all marine species in the tree were found to be active (Figure 5, solid lines). By contrast, integrase inactivation was found to be monophyletic for two soil/ freshwater subgroups, hinting at consistent selective pressure towards inactivation.

\section{Phylogenetic distribution of predicted LexA regulation and integrase functionality}

To further analyze the correlation between integrase LexA regulation and inactivation, we mapped through reciprocal BLAST searches [60] the 755 IntI homologs containing sufficient available sequence to assess both traits against the panel of IntI sequences used to reconstruct the phylogenetic tree. Even though reciprocal BLAST provides only a crude estimate of phylogenetic relationship, this mapping process allowed us to observe the apparent frequencies (Figure 5, pie charts) of both traits in the clusters represented by each tree taxon (Figure 5). Overall, the results of this analysis broadly agree with those of the ancestral-state reconstruction, and give further credence to the idea that loss of LexA regulation is associated with integrase inactivation. Nonetheless, close inspection of these results also reveals a complex pattern of phylogenetic distribution for both traits.

Among marine species, LexA regulation of intI genes is clearly prevalent, and loss of LexA regulation is only present in a few instances. One such instance is the $V$. cholerae SXT integrative-conjugative element (ICE), which harbors a class 4 integrase and for which SOSdependent transfer has been reported through an indirect path involving the phage-like SetR repressor [37,38,61]. In spite of this, mapping results show that five out of the twenty sequences clustering with the $V$. cholerae SXT integrase have predicted LexA binding sites. These sequences belong to mobile integrases in Alteromonadales species that do not have homology with the SetR transcriptional regulator, suggesting that LexA regulation may have been preserved in the absence of SetR-mediated 


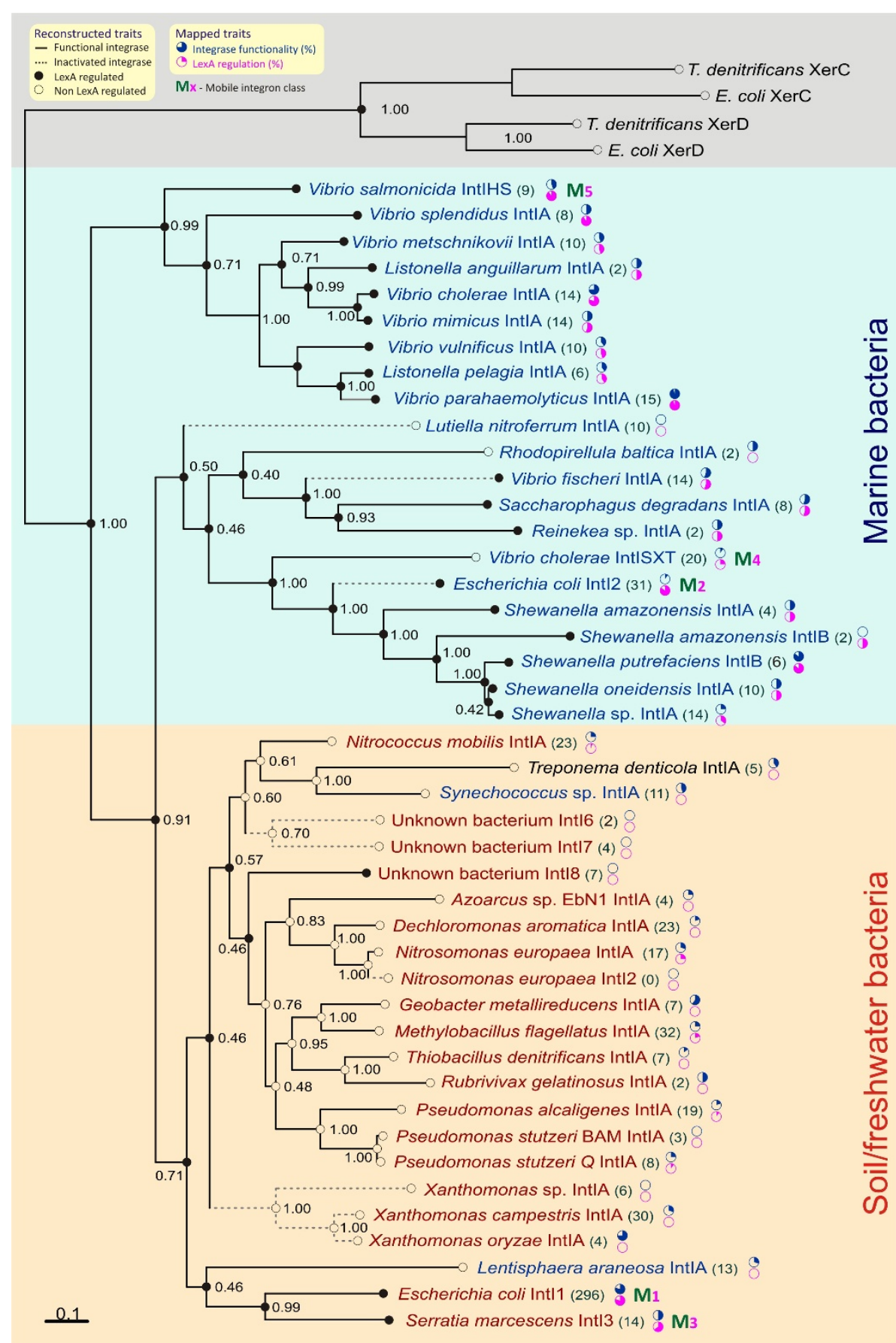

Figure 5 Phylogenetic tree of Intl protein sequences. The tree is the majority-rule consensus tree generated by MrBayes. The tree was rooted using the Escherichia coli and Thiobacillus denitrificans XerCD protein sequences as outgroup. Bayesian posterior probabilities for each branch are displayed at each branching point. Inferred states for phenotypic traits derived from parsimony ancestral-state reconstruction analysis are displayed as follows. Integrase functionality: solid lines on tree branches represent inferred integrase functionality in that branch, and dotted lines indicate non-functionality. LexA regulation: at each taxon and branching point, small filled circles represent inferred presence of LexA regulation, and open circles indicate loss of LexA regulation. For clarity, the results of maximum likelihood reconstruction are not shown (see Additional file 6 and see Additional file 7 for these). The number of sequences mapping to each taxon in the reciprocal BLAST mapping analysis is shown between brackets after the taxon name. Stacked pie charts next to this number indicate the observed percentage of integrase functionality (upper pie) and LexA regulation (lower pie) in all the analyzed integrase sequences mapping to that specific taxon. The $M$ letter followed by a subscript number $\left(M_{\chi}\right)$ legend indicates mobile integron classes (1 to 5). Background colors delineate the main division into marine and soil/ freshwater radiations and the XerCD outgroup. 
SOS regulation (see Additional file 8). Another exception is Lutiella nitroferrum, but the absence of predicted LexA sites is not surprising in a member of the Neisseriaceae, because all the sequenced members of this family lack a lexA gene [27]. A similar reasoning applies to another exception, Rhodopirellula baltica, because it is known that the LexA of Planctomycetes does not recognize the conventional E. coli LexA binding motif [27].

Conversely, loss of LexA regulation seems to be the norm among soil and freshwater species harboring chromosomal integrons. In most cases, this loss of regulation has an obvious explanation. Some families, such as the Nitrosomonadaceae and the Chromatiaceae, simply do not possess any LexA homologs, Thus explaining the absence of any LexA binding sites upstream of their intI genes [27]. A similar argument can be made for the Xanthomonadaceae, in which neither of the two identified LexA proteins recognizes the $\beta / \gamma$-Proteobacteria LexA binding motif [54], and for the Spirochetes, the $\delta$-Proteobacteria and the Cyanobacteria, in which LexA also recognizes divergent motifs $[48,62,63]$. However, reciprocal BLAST mapping indicates that there is residual LexA regulation persisting within several of these groups. The M. flagellatus cluster, for instance, has six out of thirty-two mapped sequences with predicted LexA binding sites. Careful examination reveals that, in this and all other cases of residual LexA regulation of soil/freshwater bacterial integrons, regulated integrases turn out to be harbored by a $\beta / \gamma$-Proteobacteria species or originate from environmental samples (see Additional file 9). This strongly suggests that, for the most part, LexA regulation is positively maintained when a suitable genomic background (a compatible lexA gene encoding a repressor that recognizes the $\beta / \gamma$-Proteobacteria motif) is available.

Several factors explain partly the absence of LexA binding motifs, other than the $\beta / \gamma$-Proteobacteria motif, regulating integron integrases. An obvious explanation is the lack of evolutionary time to develop such motifs. This is manifestly true for many mobile integrons subject to lateral gene transfer. Indeed, predicted $\beta / \gamma$-Proteobacteria LexA binding sites can still be seen in the mobile integrons harbored by species from distant phyla, such as the Actinobacteria. Integrase inactivation is another mechanism that several groups, such as those of the Xanthomonadales, seem to have evolved to compensate for unregulated integrase expression [16]. Even though this constitutes a general trend (Figure 4) and functionality can be temporarily restored through nonnative recombination, the observed correlation is moderate (Pearson $r=0.58^{* * * *}$ ). Moreover, integrase functionality has been assayed experimentally in several soil/freshwater chromosomal integrons in which the integrase is clearly not regulated $[64,65]$, suggesting that additional mechanisms must be at play.

Class 1 and 3 mobile integron integrases depart sharply from the trend towards loss of LexA regulation that is seen among soil/freshwater integrons. Reciprocal BLAST mapping supports the results of ancestral-state reconstruction methods, providing ample support for the persistence of LexA regulation in these well-sampled mobile integron classes. In addition, the high percentages of LexA regulation seen in both these integron classes ( $81 \%$ and $64 \%$, respectively) are consistent with high percentages of predicted regulation in the marine mobile integrons of classes 2 and 5 (84\% and 89\%, respectively; Figure 5). Beyond its fundamental relevance to bacterial adaptation, the high prevalence of predicted LexA regulation of mobile integron integrases has serious clinical implications, as it establishes a generic system for genetic interchange under control of a general stress response shared by a large group of human and animal pathogens. Furthermore, bacterial conjugation has been shown recently to induce the SOS response, triggering integrase-mediated cassette recombination, in recipient bacteria [66]. In this setting, it is important to note that integron cassettes encoding resistance to several antibiotics known to induce the SOS response, such as trimethoprim, quinolones and $\beta$-lactams, are common today $[5,67]$. This suggests that the indirect triggering effect of these antibiotics on the capture of resistance cassettes may have resulted in a very efficient selection mechanism.

A less obvious consequence of integrase SOS regulation in clinically relevant mobile integrons is its repercussion on antibiotic-resistance policies. Current policies rely largely on the detrimental effects that most resistance mechanisms inflict on bacteria, which eventually lead to loss of resistance genes in the absence of antibiotic exposure [68]. Because most cassettes are promoterless, the most ancient cassettes (located at the distal part of the integron) are subject to severe polar effects, leading to rare or non-existent protein products (Figure 1) [4]. In this context, the incorporation of SOS regulation in integrons puts forward a mechanism by which antibioticresistance genes and other useful adaptations can be silently set aside, while current adaptive traits are maintained. In time of stress, such as exposure to antibiotics, the relevant resistance cassettes can be called upon by integrase-mediated translocation, and thus selected for only when their expression is required. Furthermore, the cassette genes that have been temporarily relegated to distal positions in integrons may also sustain increased evolution rates, generating a substantial pool of variability from which to draw on when the appropriate selective pressures resurface [69]. 
Reciprocal BLAST mapping also shows that predicted integrase inactivation is very common among soil/freshwater bacteria, coinciding with a prevailing loss of putative LexA regulation. Nonetheless, predicted integrase inactivation is also relatively common in marine species. Even though the predicted integrase inactivation correlates well with reduced LexA regulation (Figure 4), there are notable outliers to this trend in both radiations. For instance, among mobile class 5 integrons, only $44 \%$ of mapped integrases seem to be functional, despite predicted LexA regulation in $89 \%$ of them. The opposite is also true; many mobile integrons with putatively functional integrases have disrupted, absent or non-native LexA binding sites. This suggests that lack of LexA regulation can be tolerated or selected for when it provides adaptive benefit. We have shown here that in some mobile class 1 integrons, the LexA binding site has been disrupted by a GGG insertion that drastically increases the expression of antibioticresistance cassettes (Figure 3). In a similar vein, it seems likely that sustained integrase activity (with its associated shuffling of gene cassettes $[21,25]$ ) must be preferable to permanent inactivation under the shifting selective environments associated with clinical environments and mobile integrons. This would explain why integrase inactivation is not seen as frequently in mobile integron classes associated with clinical settings, in spite of their dissemination into bacterial species that do not harbor a lexA gene capable of regulating the preset LexA binding site.

Overall, however, the pattern of integrase inactivation is broadly in agreement with that reported previously [53]. In fact, we found a higher proportion (46\%) of inactivated IntI proteins than that reported previously [53], indicating that integrase inactivation is a pervasive phenomenon and typically correlated with loss of LexA regulation. Hence, our findings suggest that putative integrase inactivation is the main mechanism evolved to deal with lack of LexA regulation, but it seems likely that other factors must provide heightened tolerance to unregulated integrase activity in soil/freshwater bacteria. Smaller integron sizes and lessened integrase activity may both contribute to make unregulated integrase expression more tolerable, but regulation by an alternative transcription factor is an obvious possibility that needs to be carefully explored. This is particularly true because most integrase functionality assays have been carried out in a non-native context $[64,65]$ and may thus have missed regulatory effects. The quest to define precisely the multiple mechanisms behind this adaption is an important goal, because the lack of a mechanism to mitigate the effects of integrase activity upon loss of LexA regulation may well lie at the root of the intriguing absence of chromosomal integrons from many bacterial phyla [53].

\section{Conclusions}

The results presented here illustrate the extent of SOS regulation of integron integrases, and provide several important clues to the evolution of this regulation and to the evolution of bacterial integrons. The combination of in silico and in vivo assays allows us to conclude that LexA regulation was probably present in the primordial integron and that its loss may be linked to a number of factors, including inactivation of the integrase gene and enhancement of resistance cassettes expression. Our findings have important clinical implications for the evolution of antibiotic resistance, and suggest that the emergence of mechanisms to palliate unregulated integrase expression may provide an explanation for the uneven distribution of integrons across the Bacteria domain.

\section{Methods}

\section{Data mining and preprocessing}

A custom set of scripts was developed in BioPhyton to search for intI homologs on NCBI GenBank databases (NR, ENV and WGS). The scripts retrieved and reannotated both the intI coding sequences and their corresponding upstream sequences. The scripts used the whole VchIntIA protein sequence (AAC38424) and its IntI specific domain [70] (positions 186 to 245 in VchIntIA) as a query for a TBLASTN search. To limit the number of false positives, a cut-off e-value of $10^{-5}$ was set, and only sequences matching both queries were retrieved.

TBLASTN results were used to identify frameshift and deletion events of up to $100 \mathrm{bp}$. Larger events where not considered. The nucleotide sequences spanning the full length of the processed hits and $1 \mathrm{~kb}$ upstream of the hit start were recovered. Conceptual translations of these sequences (corrected for frameshift when necessary) were then used to search a curated reference panel using BLASTP. The reference panel comprised 43 phylogenetically diverse IntI proteins, phage integrases and XerCD recombinases. The reference sequence of the best reciprocal hit was used to consistently re-annotate the start and stop points of all retrieved sequences, thereby allowing homogenization of the dataset and efficient detection of in-frame premature stop codons. Sequences with a best reciprocal hit not belonging to the IntI family (that is, phage integrases and XerCD recombinases) were removed from further analysis. Similarly, all IntI homologs lacking a significant amount of coding sequence at both ends of the predicted coding region ( +30 bp downstream of the start codon and -90 bp upstream of stop codon) were also removed from further analysis. Duplicates resulting from the use of partially redundant databases were removed, defining duplicates as two sequences having the same 
sequences, coordinates and NCBI taxonomical assignment, and the same strain or plasmid number when applicable. The final annotated dataset comprised 1,483 sequences, and is available online as supplementary material in both GenBank (.GBK) and spreadsheet-compatible (.XLS) format (see Additional file 10, see Additional file 11).

\section{Assessment of protein functionality}

Integrase functionality was assessed systematically using a custom rule-based system operating on aligned IntI sequences. To generate functional rules to detect inactivation, we analyzed published structural and mutational studies of both the chromosomal $V$. cholerae IntI4 and the mobile IntI1 integron integrases [22,70-74]. From this analysis, we identified a list of five essential residues in the catalytic site that cannot be mutated (R135, K160, H267, R270, H293, Y302; positions relative to the $V$. cholerae IntIA sequence), and eight residues essential for binding, for which only a limited range of substitutions is likely to be tolerated (L202 $(\rightarrow$ LIVM), P203 $(\rightarrow$ PST), K209, Y210 $(\rightarrow$ YFWH), P211 $(\rightarrow$ PRQ), R239 $(\rightarrow \mathrm{KRH}), \mathrm{H} 240(\rightarrow \mathrm{KRH}), \mathrm{H} 241(\rightarrow \mathrm{KRH})$; positions again relative to the $V$. cholerae IntIA sequence).

A multiple alignment of all IntI sequences in the reference panel was generated using MUSCLE software http://www.drive5.com/muscle/ with an opening gap penalty of -20 , and otherwise standard parameters [75]. This alignment was used to propagate the functional rules defined on the VchIntIA sequence towards the reference panel IntI sequences. The consistency of this propagation was reviewed manually. Pairwise alignments of all the TBLASTN identified homologs with their corresponding best hits were used to further propagate the functional model and allow a decision on whether each particular protein should be considered functional. IntI sequences containing an internal stop, a frameshift and/ or any number of inactivating mutations were tagged as 'non-functional'. If either the start or stop of sequence was unavailable (see above), the functionality of the corresponding protein was tagged as 'unknown'. Otherwise, the protein was considered functional by default.

The automated rule-based system was evaluated against a reference set of integron integrase sequences for which activity has been experimentally assessed [64,65,76-80]. This reference set encompasses active and inactive integrases from both marine and soil/freshwater chromosomal integrons, and class 1, 2 and 3 mobile integrons. The rule-based system was able to correctly predict integrase activity in all these cases. In addition, it also detected all indels, frameshift and nonsense mutations that have been reported previously in independent studies as leading to integrase inactivation $[16,53]$.

\section{In silico searches for LexA binding sites}

The presence of LexA binding sites on all the retrieved int I homolog sequences was assessed by scanning them using xFITOM http://compbio.umbc.edu/2280/, a generic program for binding site search in genomic sequences $[81,82]$. Searches were conducted using the $R_{i}$ index [83] and a motif-normalized threshold as reported previously [84]. Identified sites were considered ' $w /$ functional box' if located within -100 or $+50 \mathrm{bp}$ of the reannotated intI start codon. When the sequence in the specified range was not fully available, this feature was tagged as 'unknown'. Searches were conducted using the 15 different LexA binding motifs reported to date [27], which include those of largely sampled phylogenetic groups, such as the Firmicutes, the Actinobacteria, the Cyanobacteira or the Alpha Proteobacteria [62,85-87]. We also identified, and specifically searched for, a particular motif consisting of a LexA binding site inactivated by the insertion of a GGG triplet. These sites are referred to as 'broken', and were categorized as 'without functional box'. The results of integrase functionality and LexA binding site searches are fully annotated on the main dataset files (see Additional file 10, see Additional file 11).

\section{Phylogenetic analyses}

Alignments of the reference-panel protein sequences were carried out using a combined procedure to improve alignment quality as described previously [88]. Protein sequences were first aligned with CLUSTALW (version1.83; http://www.ebi.ac.uk/Tools/msa/clustalw2/[89] using Gonnet matrices and default [10], twenty-five and five gap-opening penalties for the multiple alignment stage, thus generating three different alignments. These three different alignments, together with a local alignment generated by the T-COFFEE Lalign method, were integrated as libraries into T-COFFEE (version 1.37; http://www.ebi.ac.uk/Tools/msa/tcoffee/[90] for optimization. The optimized alignment was then processed with Gblocks (version 0.91b; http://molevol.cmima.csic. es/castresana/Gblocks.html[91] with the half-gaps setting and otherwise default parameters to select conserved positions and discard poorly aligned and phylogenetically unreliable information. Phylogenetic analyses were then carried out using MrBayes (version 3.1.1; http://mrbayes. csit.fsu.edu/ and PHYML version 2.4.1; http://code.google.com/p/phyml/[92] for Bayesian inference of tree topologies as reported previously [88]. A mixed fourcategory $\gamma$ distributed rate plus proportion of invariable sites model [invgamma] was applied and its parameters were estimated independently by the program. Eight independent MrBayes Metropolis-Coupled Markov Chain Monte Carlo runs were carried out with four 
independent chains for $10^{6}$ generations. The resulting phylogenetic trees were plotted with TreeView (version 1.6.6; http://taxonomy.zoology.gla.ac.uk/rod/treeview. html[93] and edited for presentation using CorelDraw Graphic Suite (version 12; Corel Corp., Fremont, CA, USA).

Ancestral-state reconstruction was conducted with the Mesquite ancestral-state reconstruction package (Mesquite Software Inc., Austin, TX, USA) [94] using the majority-rule consensus tree generated by MrBayes. The results of in silico searches for LexA binding sites were mapped into a discrete $(1 / 0 /$ ?) character for each taxon of the tree. Reconstruction of LexA binding site presence was first carried out using the ML reconstruction method $[95,96]$ and the AsymmMk model (Asymmetrical Markov $\mathrm{k}$-state two-parameter model), estimating asymmetric rates of change between characters. The estimated rates (0.145 forward, 0.813 backward) were then converted into parsimony steps by direct inversion $(6.89,1.23)$, and used to generate the step matrix for parsimony reconstruction [97]. The results from in silico integrase functionality assessment were also mapped into a discrete (1/0/?) character for each taxon. Ancestral-state reconstruction for this character was carried out using both an ordered parsimony model and AsymmMk-based maximum-likelihood model. The results of both reconstruction methods were broadly in agreement (see Additional file 7), but for clarity, only parsimony results are superimposed on Figure 5.

\section{EMSA}

The $V$. parahaemolyticus and E. coli lexA genes were amplified using suitable primers (see Additional file 12) and cloned into a pET15b vector (see Additional file 13). Overexpression and purification of the corresponding LexA protein was performed as described previously for other LexA proteins [84]. Each DNA probe was constructed using two complementary 100 bp synthetic oligonucleotides (see Additional file 12). EMSA experiments were performed as described previously [84], using 80 $\mathrm{nmol} / \mathrm{l} \mathrm{V}$. parahaemolyticus LexA or $200 \mathrm{nmol} / \mathrm{l}$ of E.coli LexA protein and $20 \mathrm{ng}$ of each DIG-marked DNA probe in the binding mixture. For EMSA competitive assays, 200 fold of either specific or non-specific non-labeled DNA was added to the binding mixture. In all cases, samples were loaded onto $6 \%$ non-denaturing Tris-glycine polyacrylamide gels. Digoxigenin-labeled DNA-protein complexes were detected using the manufacturer's protocol (Roche Applied Science, Indianapolis, IN, USA).

\section{RNA extraction and RT-PCR}

RT-PCR experiments were performed (Titan One Tube RT-PCR System; Roche) with suitable oligonucleotides (see Additional file 12 for list), following the manufacturer's instructions. Real-time quantitative RT-PCR analysis of total RNA was carried out in a PCR system, (LightCycler; Roche), using a commercial kit (LCRNA Master SYBR Green I Kitl Roche) according to the manufacturer's instructions. Transcription of pMUR050 intID1 and intID2 genes (under control of $P_{\text {intII }}{ }^{-}$and $P_{\text {intII }}{ }^{+}$, respectively) was determined in wild-type $E$. coli $\mathrm{K} 12$ and in a lexA-defective strain (UA6189). Both strains contained either the pUA1105 (intA1) or the pUA1106 (intA2) plasmid. Expression of the V. parahaemolyticus intI gene was tested in the ATCC17802 wild-type strain and in a lexA-defective strain (UA10001) (see Additional file 13). In both cases, expression of the $r e c A$ gene was used as the positive control, and the mRNA concentration for each gene was normalized to that of the housekeeping $d x s$ gene. The expression factor was calculated as the ratio of the relative mRNA concentration for each gene in the corresponding lexA mutant strain with respect to that in the wild type. In each case, the mean value from three independent experiments (each in triplicate) was calculated. Strains UA6189 and UA10001 were constructed, respectively, using the Lambda-Red recombinase system [98] or the marker exchange procedure, as described previously [99].

\section{Funding}

This work wmas supported by grants from the Ministère de la Recherche et de l'Enseignement supérieur, the Conseil Régional du Limousin, the Fondation pour la Recherche Médicale (FRM) and from the Institut National de la Santé et de la Recherche Médicale (Inserm) for the Ploy laboratory; by the Institut Pasteur, the Centre National de la Recherche Scientifique (CNRS URA 2171), the FRM and the EU (NoE EuroPathoGenomics, LSHB-CT-2005-512061), for the Mazel laboratory; and by grants BFU2008-01078/BMC from the Ministerio de Ciencia e Innovación de España and 2009SGR-1106 from the Generalitat de Catalunya, for the Barbé laboratory. NSA was supported by the Fundació Cellex at the Erill laboratory.

\section{Additional material}

Additional file 1: Identified LexA binding sites in the promoter region $(-100,+50$ of the start codon) of integrase homologs from the WGS, NR and ENV NCBI databases.

Additional file 2: List of the 93 unique, distinct LexA binding sites identified in this work.

Additional file 3: (A) Schematic representation of the pMUR050 plasmid, showing (bold) the location of the two int/1 homologs. (B) Schematic representation of the promoter region of both int/1 homologs, showing the organization of the $P_{\text {int } / 1}{ }^{-}$and $P_{\text {int } / 1}{ }^{+}$promoters, the standard cassette promoter $\left(P_{C}\right.$ and the secondary cassette promoter $\left(P_{C 2}\right)$ enabled by the GGG insertion. For both genes, promoter elements are also

mapped into their corresponding sequence fragments. Red boxes depict LexA binding sites, black boxes outline the -35 and -10 elements of the $P_{\text {int/ }}$ promoter, and green boxes depict the secondary $P_{C 2}$ promoter. 
Additional file 4: List of the 45 LexA binding sites presenting GGG disruption identified in this work.

Additional file 5: Predicted functionality status for the 1,135 intl homolog sequences for which sufficient coding sequence was available to determine inactivation using the in silico method reported in this work.

Additional file 6: Phylogenetic tree of Intl protein sequences showing the maximum likelihood ancestral-state reconstruction of LexA regulation, as inferred from in silico analyses, using an asymmetric two-state Markov model (AsymmMk) in Mesquite [94] The tree is the majority-rule consensus tree generated by MrBayes, and was rooted using the Escherichia coli and Thiobacillus denitrificans XerCD protein sequences as outgroup. At each taxon and branching point, piefilled circles indicate the likelihood of LexA regulation at each node, with a completely filled circle indicating certainty of LexA regulation, and a completely open circle indicating certainty of lack of LexA regulation. Taxon name colors indicate the natural habitat of each organism (blue for marine, green for soil/freshwater, black for ambiguous) or their pertaining to the outgroup (red). Azo $=$ Azoarcus sp. EbN1; Dar $=$ Dechloromonas aromatica; $\mathrm{ECO}=\mathrm{E}$. coli; $\mathrm{Gme}=$ Geobacter metallireducens; Lan = Listonella anguillarum; Lar = Lentisphaera araneosa; Lni = Lutiella nitroferrum; Lpe = Listonella pelagia; Mfl = Methylobacillus flagellatus; Neu = Nitrosomonas europaea; $\mathrm{Nmo}=$ Nitrococcus mobilis; $\mathrm{Pal}=$ Pseudomonas alcaligenes; Pme $=$ Pseudomonas mendocina; $\mathrm{Ppr}=$ Photobacterium profundum; PstuBA $=$ Pseudomonas stutzeri BAM; $P$ stuQ $=$ Pseudomonas stutzeri Q; Rei = Reinekea sp.; Rba = Rhodopirellula baltica; Rge = Rubrivivax gelatinosus; Sde = Saccharophagus degradans; Sam = Shewanella amazonensis; Ssp = Shewanella sp. MR-7; Son = Shewanella oneidensis; Spu = Shewanella putrefaciens; SynSp = Synechococcus sp; Tden $=$ Treponema denticola; Tde $=$ Thiobacillus denitrificans; Vch $=$ Vibrio cholerae; $\mathrm{Vfi}=$ Vibrio fischeri; $\mathrm{Vme}=$ Vibrio metschnikovii; $\mathrm{Vmi}=$ Vibrio mimicus; $\mathrm{Vpa}=$ Vibrio parahaemolyticus; $\mathrm{Vsp}=$ Vibrio splendidus; $\mathrm{Vvu}=$ Vibrio vulnificus; $\mathrm{Xca}=$ Xanthomonas campestris; $\mathrm{Xor}=$ Xanthomonas oryzae; Xsp = Xanthomonas sp.

Additional file 7: Phylogenetic tree of Intl protein sequences showing the maximum likelihood ancestral-state reconstruction of integrase functionality, as inferred from in silico analyses, using an asymmetrical two-state Markov model (AsymmMk) in Mesquite [94]. The tree is the majority-rule consensus tree generated by MrBayes, and was rooted using the Escherichia coli and Thiobacillus denitrificans XerCD protein sequences as outgroup. At each taxon and branching point, piefilled circles indicate the likelihood of integrase functionality at each node, with a completely filled circle indicating certainty of integrase functionality and a completely open circle indicating certainty of integrase inactivation. Taxon name colors indicate the natural habitat of each organism (blue for marine, green for soil/freshwater, black for ambiguous) or their pertaining to the outgroup (red). Azo $=$ Azoarcus sp. EbN1; Dar = Dechloromonas aromatica; Eco $=$ Escherichia coli; $\mathrm{Gme}=$ Geobacter metallireducens; Lan = Listonella anguillarum; Lar = Lentisphaera araneosa; Lni = Lutiella nitroferrum; Lpe = Listonella pelagia; $\mathrm{Mfl}=$ Methylobacillus flagellatus; $\mathrm{Neu}=$ Nitrosomonas europaea; $\mathrm{Nmo}=$ Nitrococcus mobilis; $\mathrm{Pal}=$ Pseudomonas alcaligenes; $P$ me $=$ Pseudomonas mendocina; $\mathrm{Ppr}=$ Photobacterium profundum; $\mathrm{PstuBA}=$ Pseudomonas stutzeri $\mathrm{BAM} ; \mathrm{PstuQ}=$ Pseudomonas stutzeri $\mathrm{Q} ;$ Rei $=$ Reinekea sp.; $\mathrm{Rba}=$ Rhodopirellula baltica; $\mathrm{Rge}=$ Rubrivivax gelatinosus; $\mathrm{Sde}=$ Saccharophagu degradans; Sam = Shewanella amazonensis; $\mathrm{Ssp}=$ Shewanella sp. MR-7; Son = Shewanella oneidensis; Spu = Shewanella putrefaciens; SynSp = Synechococcus sp; Tden $=$ Treponema denticola; Tde $=$ Thiobacillus denitrificans; $\mathrm{Vch}=$ Vibrio cholerae; $\mathrm{Vfi}=$ Vibrio fischeri; $\mathrm{Vme}=$ Vibrio metschnikovii; $\mathrm{Vmi}=$ Vibrio mimicus; $\mathrm{Vpa}=$ Vibrio parahaemolyticus; $\mathrm{Vsp}=$ Vibrio splendidus; $\mathrm{Vvu}=$ Vibrio vulnificus; $\mathrm{Xca}=$ Xanthomonas campestris; Xor $=$ Xanthomonas oryzae; $\mathrm{Xsp}=$ Xanthomonas $\mathrm{sp}$.

Additional file 8: List of sequences mapping to the VchlntISXT (AAK95987) taxon according to reciprocal BLAST results. Sequences belonging to the Alteromonadales order are shown yellow.

Additional file 9: Sequences from soil/freshwater bacteria clusters showing evidence of residual LexA regulation. Sequences with identified LexA binding sites are shown in yellow.
Additional file 10: Complete and fully annotated set of IntI homologs identified in this work in GenBank format.

Additional file 11: Complete and fully annotated set of Intl homologs identified in this work in Excel (XLS) format.

Additional file 12: Oligonucleotides used in this work.

Additional file 13: Strains and plasmids used in this work.

Additional file 14: Statistical summary of reciprocal BLAST mapping results with regard to the two in silico predicted traits analyzed here: LexA regulation and integrase functionality.

\section{Acknowledgements}

We thank Mike C. O'Neill for his careful reading and comments on the different versions of this manuscript. We also thank Nicholas Friedman for his assistance in ancestral-state reconstruction techniques.

\section{Author details}

'Institut Pasteur, Unité Plasticité du Génome Bactérien, CNRS URA 2171، 75015 Paris, France. ${ }^{2}$ Departament de Genètica i de Microbiologia, Universitat Autònoma de Barcelona, 08193 Bellaterra, Spain. ${ }^{3}$ Department of Biological Sciences, University of Maryland Baltimore County, Baltimore 21228, USA. "Université de Limoges, Faculté de Médecine, EA3175, INSERM, Equipe Avenir, Limoges 87000, France. ${ }^{5}$ Departamento de Sanidad Animal, Facultad de Veterinaria, and VISAVET, Universidad Complutense de Madrid, 28040 Madrid, Spain.

\section{Authors' contributions}

GC implemented the functionality assessment method and carried out data mining, preprocessing and statistical analysis. NSA performed protein purification, RT-PCR and mobility-shift assays. SC and JB designed and directed the in vitro and in vivo studies and provided expertise on LexA binding motifs. EG, SDR and MCP coordinated in vitro and in vivo analyses and provided expertise on integrase expression. BGZ provided the pMUR plasmid and participated in coordination. GC, NSA, DM and IE conceived of the study and participated in its design and coordination. IE developed the site-search method, carried out phylogenetic reconstruction and ancestralstate reconstruction, and directed the in silico and statistical analyses. DM and GC developed the functionality assessment method. DM and IE coordinated and directed this work and drafted the manuscript. All authors read and approved the final manuscript.

\section{Competing interests}

The authors declare that they have no competing interests.

Received: 9 November 2010 Accepted: 30 April 2011

Published: 30 April 2011

\section{References}

1. Stokes HW, Hall RM: A novel family of potentially mobile DNA elements encoding site-specific gene-integration functions: integrons. Molecular microbiology 1989, 3:1669-1683.

2. Collis CM, Kim MJ, Stokes HW, Hall RM: Integron-encoded Intl integrases preferentially recognize the adjacent cognate attl site in recombination with a 59-be site. Molecular microbiology 2002, 46:1415-1427.

3. Levesque C, Brassard S, Lapointe J, Roy PH: Diversity and relative strength of tandem promoters for the antibiotic-resistance genes of several integrons. Gene 1994, 142:49-54.

4. Collis CM, Hall RM: Expression of antibiotic resistance genes in the integrated cassettes of integrons. Antimicrobial agents and chemotherapy 1995, 39:155-162.

5. Rowe-Magnus DA, Mazel D: The role of integrons in antibiotic resistance gene capture. Int J Med Microbiol 2002, 292:115-125.

6. Fluit AC, Schmitz FJ: Resistance integrons and super-integrons. Clin Microbiol Infect 2004, 10:272-288.

7. Partridge SR, Tsafnat G, Coiera E, Iredell JR: Gene cassettes and cassette arrays in mobile resistance integrons. FEMS microbiology reviews 2009, 33:757-784. 
8. Cambray G, Guerout AM, Mazel D: Integrons. Annual review of genetics 2010, 44:141-166.

9. Mazel D: Integrons: agents of bacterial evolution. Nature reviews 2006, 4:608-620.

10. Boucher Y, Labbate M, Koenig JE, Stokes HW: Integrons: mobilizable platforms that promote genetic diversity in bacteria. Trends in microbiology 2007, 15:301-309.

11. Mazel D, Dychinco B, Webb VA, Davies J: A distinctive class of integron in the Vibrio cholerae genome. Science (New York, NY) 1998, 280:605-608.

12. Rowe-Magnus DA, Guerout AM, Mazel D: Super-integrons. Research in microbiology 1999, 150:641-651.

13. Rowe-Magnus DA, Guerout AM, Ploncard P, Dychinco B, Davies J, Mazel D: The evolutionary history of chromosomal super-integrons provides an ancestry for multiresistant integrons. Proceedings of the National Academy of Sciences of the United States of America 2001, 98:652-657.

14. Vaisvila R, Morgan RD, Posfai J, Raleigh EA: Discovery and distribution of super-integrons among pseudomonads. Molecular microbiology 2001, 42:587-601.

15. Rowe-Magnus DA, Guerout AM, Biskri L, Bouige P, Mazel D: Comparative analysis of superintegrons: engineering extensive genetic diversity in the Vibrionaceae. Genome research 2003, 13:428-442.

16. Gillings MR, Holley MP, Stokes HW, Holmes AJ: Integrons in Xanthomonas: a source of species genome diversity. Proceedings of the National Academy of Sciences of the United States of America 2005, 102:4419-4424.

17. Rowe-Magnus DA, Guerout AM, Mazel D: Bacterial resistance evolution by recruitment of super-integron gene cassettes. Molecular microbiology 2002, 43:1657-1669.

18. Melano R, Petroni A, Garutti A, Saka HA, Mange L, Pasteran F, Rapoport M, Rossi A, Galas M: New carbenicillin-hydrolyzing beta-lactamase (CARB-7) from Vibrio cholerae non-O1, non-0139 strains encoded by the VCR region of the $\mathrm{V}$. cholerae genome. Antimicrobial agents and chemotherapy 2002, 46:2162-2168.

19. Petroni A, Melano RG, Saka HA, Garutti A, Mange L, Pasteran F, Rapoport M, Miranda M, Faccone D, Rossi A, Hoffman PS, Galas MF: CARB-9, a carbenicillinase encoded in the VCR region of Vibrio cholerae non-O1, non-0139 belongs to a family of cassette-encoded beta-lactamases. Antimicrobial agents and chemotherapy 2004, 48:4042-4046.

20. Le Roux F, Zouine M, Chakroun N, Binesse J, Saulnier D, Bouchier C, Zidane N, Ma L, Rusniok C, Lajus A, Buchrieser C, Médigue C, Polz MF, Mazel D: Genome sequence of Vibrio splendidus: an abundant planctonic marine species with a large genotypic diversity. Environmental microbiology 2009, 11:1959-1970.

21. Labbate M, Boucher Y, Joss MJ, Michael CA, Gillings MR, Stokes HW: Use of chromosomal integron arrays as a phylogenetic typing system for Vibrio cholerae pandemic strains. Microbiology (Reading, England) 2007, 153:1488-1498.

22. MacDonald D, Demarre G, Bouvier M, Mazel D, Gopaul DN: Structural basis for broad DNA-specificity in integron recombination. Nature 2006, 440:1157-1162.

23. Bouvier M, Ducos-Galand M, Loot C, Bikard D, Mazel D: Structural features of single-stranded integron cassette attC sites and their role in strand selection. PLoS genetics 2009, 5:e1000632.

24. Bouvier M, Demarre G, Mazel D: Integron cassette insertion: a recombination process involving a folded single strand substrate. The EMBO journal 2005, 24:4356-4367.

25. Guerin E, Cambray G, Sanchez-Alberola N, Campoy S, Erill I, Da Re S, Gonzalez-Zorn B, Barbe J, Ploy M-C, Mazel D: The SOS Response Controls Integron Recombination. Science 2009, 324:1034.

26. Walker GC: Mutagenesis and inducible responses to deoxyribonucleic acid damage in Escherichia coli. Microbiol Rev 1984, 48:60-93.

27. Erill I, Campoy S, Barbe J: Aeons of distress: an evolutionary perspective on the bacterial SOS response. FEMS microbiology reviews 2007, 31:637-656.

28. Aertsen A, Michiels CW: Upstream of the SOS response: figure out the trigger. Trends in microbiology 2006, 14:421-423.

29. Sassanfar M, Roberts JW: Nature of the SOS-inducing signal in Escherichia coli. The involvement of DNA replication. Journal of molecular biology 1990, 212:79-96.

30. Little JW: Mechanism of specific LexA cleavage: autodigestion and the role of RecA coprotease. Biochimie 1991, 73:411-421.
31. Fernandez De Henestrosa AR, Ogi T, Aoyagi S, Chafin D, Hayes JJ, Ohmori $\mathrm{H}$, Woodgate R: Identification of additional genes belonging to the LexA regulon in Escherichia coli. Molecular microbiology 2000, 35:1560-1572.

32. Waldor MK, Friedman DI: Phage regulatory circuits and virulence gene expression. Current opinion in microbiology 2005, 8:459-465.

33. Quinones M, Davis BM, Waldor MK: Activation of the Vibrio cholerae SOS response is not required for intestinal cholera toxin production or colonization. Infection and immunity 2006, 74:927-930.

34. Kimmitt PT, Harwood CR, Barer MR: Toxin gene expression by shiga toxinproducing Escherichia coli: the role of antibiotics and the bacterial SOS response. Emerging infectious diseases 2000, 6:458-465.

35. Aleshkin Gl, Kadzhaev KV, Markov AP: High and low UV-dose responses in SOS-induction of the precise excision of transposons tn1, Tn5 and Tn10 in Escherichia coli. Mutation research 1998, 401:179-191.

36. Ubeda C, Maiques E, Knecht E, Lasa I, Novick RP, Penades JR: Antibioticinduced SOS response promotes horizontal dissemination of pathogenicity island-encoded virulence factors in staphylococci. Molecular microbiology 2005, 56:836-844.

37. Kelley WL: Lex marks the spot: the virulent side of SOS and a closer look at the LexA regulon. Molecular microbiology 2006, 62:1228-1238.

38. Beaber JW, Hochhut B, Waldor MK: SOS response promotes horizontal dissemination of antibiotic resistance genes. Nature 2004, 427:72-74

39. Phillips I, Culebras E, Moreno F, Baquero F: Induction of the SOS response by new 4-quinolones. The Journal of antimicrobial chemotherapy 1987, 20:631-638.

40. Miller C, Thomsen LE, Gaggero C, Mosseri R, Ingmer H, Cohen SN: SOS response induction by beta-lactams and bacterial defense against antibiotic lethality. Science (New York, NY) 2004, 305:1629-1631.

41. Maiques E, Ubeda C, Campoy S, Salvador N, Lasa I, Novick RP, Barbe J, Penades JR: beta-lactam antibiotics induce the SOS response and horizontal transfer of virulence factors in Staphylococcus aureus. Journal of bacteriology 2006, 188:2726-2729.

42. Goerke C, Koller J, Wolz C: Ciprofloxacin and trimethoprim cause phage induction and virulence modulation in Staphylococcus aureus. Antimicrobial agents and chemotherapy 2006, 50:171-177.

43. Avison MB: New approaches to combating antimicrobial drug resistance. Genome biology 2005, 6:243.

44. Michael CA, Gillings MR, Holmes AJ, Hughes L, Andrew NR, Holley MP, Stokes HW: Mobile gene cassettes: a fundamental resource for bacterial evolution. The American naturalist 2004, 164:1-12.

45. Tapias A, Barbe J: Mutational analysis of the Rhizobium etli recA operator. Journal of bacteriology 1998, 180:6325-6331.

46. Campoy S, Fontes M, Padmanabhan S, Cortes P, Llagostera M, Barbe J: LexA-independent DNA damage-mediated induction of gene expression in Myxococcus xanthus. Molecular microbiology 2003, 49:769-781.

47. Mazon G, Erill I, Campoy S, Cortes P, Forano E, Barbe J: Reconstruction of the evolutionary history of the LexA-binding sequence. Microbiology 2004, 150:3783-3795.

48. Jara M, Nunez C, Campoy S, Fernandez de Henestrosa AR, Lovley DR, Barbe J: Geobacter sulfurreducens has two autoregulated lexA genes whose products do not bind the recA promoter: differing responses of lexA and recA to DNA damage. Journal of bacteriology 2003, 185:2493-2502.

49. Campoy S, Salvador N, Cortes P, Erill I, Barbe J: Expression of canonical SOS genes is not under LexA repression in Bdellovibrio bacteriovorus. Journal of bacteriology 2005, 187:5367-5375.

50. Kim T-E, Kwon H-J, Cho S-H, Kim S, Lee B-K, Yoo H-S, Park Y-H, Kim S-J: Molecular differentiation of common promoters in Salmonella class 1 integrons. Journal of Microbiological Methods 2007, 68:453-457.

51. Jove T, Da Re S, Denis F, Mazel D, Ploy MC: Inverse correlation between promoter strength and excision activity in class 1 integrons. PLoS genetics 2010, 6:e1000793.

52. Gonzalez-Zorn B, Catalan A, Escudero JA, Dominguez L, Teshager $T$, Porrero C, Moreno MA: Genetic basis for dissemination of armA. The Journal of antimicrobial chemotherapy 2005, 56:583-585.

53. Nemergut DR, Robeson MS, Kysela RF, Martin AP, Schmidt SK, Knight R: Insights and inferences about integron evolution from genomic data. BMC genomics 2008, 9:261. 
54. Yang MK, Yang YC, Hsu CH: Characterization of Xanthomonas axonopodis pv. citri LexA: recognition of the LexA binding site. Mol Genet Genomics 2002, 268:477-487.

55. Campoy S, Mazon G, Fernandez de Henestrosa AR, Llagostera M, Monteiro PB, Barbe J: A new regulatory DNA motif of the gamma subclass Proteobacteria: identification of the LexA protein binding site of the plant pathogen Xylella fastidiosa. Microbiology (Reading, England) 2002, 148:3583-3597.

56. Diaz-Mejia JJ, Amabile-Cuevas CF, Rosas I, Souza V: An analysis of the evolutionary relationships of integron integrases, with emphasis on the prevalence of class 1 integrons in Escherichia coli isolates from clinical and environmental origins. Microbiology (Reading, England) 2008, 154:94-102

57. Boucher Y, Nesbo CL, Joss MJ, Robinson A, Mabbutt BC, Gillings MR, Doolittle WF, Stokes HW: Recovery and evolutionary analysis of complete integron gene cassette arrays from Vibrio. BMC Evolutionary Biology 2006, 6:3.

58. Larouche A, Roy PH: Analysis by mutagenesis of a chromosomal integron integrase from Shewanella amazonensis SB2BT. Journal of bacteriology 2009, 191:1933-1940.

59. Gillings $M$, Boucher $Y$, Labbate $M$, Holmes A, Krishnan S, Holley M, Stokes HW: The evolution of class 1 integrons and the rise of antibiotic resistance. Journal of bacteriology 2008, 190:5095-5100.

60. Fuchsman CA, Rocap G: Whole-genome reciprocal BLAST analysis reveals that planctomycetes do not share an unusually large number of genes with Eukarya and Archaea. Applied and environmental microbiology 2006, 72:6841-6844.

61. Beaber JW, Waldor MK: Identification of operators and promoters that control SXT conjugative transfer. Journal of bacteriology 2004, 186:5945-5949.

62. Mazon G, Lucena JM, Campoy S, Fernandez de Henestrosa AR, Candau P, Barbe J: LexA-binding sequences in Gram-positive and cyanobacteria are closely related. Mol Genet Genomics 2004, 271:40-49.

63. Cune J, Cullen P, Mazon G, Campoy S, Adler B, Barbe J: The Leptospira interrogans lexA gene is not autoregulated. Journal of bacteriology 2005, 187:5841-5845.

64. Holmes AJ, Holley MP, Mahon A, Nield B, Gillings M, Stokes HW Recombination activity of a distinctive integron-gene cassette system associated with Pseudomonas stutzeri populations in soil. Journal of bacteriology 2003, 185:918-928.

65. Leon G, Roy PH: Excision and integration of cassettes by an integron integrase of Nitrosomonas europaea. Journal of bacteriology 2003, 185:2036-2041

66. Baharoglu Z, Bikard D, Mazel D: Conjugative DNA transfer induces the bacterial SOS response and promotes antibiotic resistance development through integron activation. PLOS genetics 2010, 6:e1001165.

67. Fonseca EL, Dos Santos Freitas F, Vieira W, Vicente AC: New qnr Gene Cassettes Associated with Superintegron Repeats in Vibrio cholerae 01. Emerging infectious diseases 2008, 14:1129-1131.

68. Andersson DI, Levin BR: The biological cost of antibiotic resistance. Current opinion in microbiology 1999, 2:489-493.

69. Gupta RD, Tawfik DS: Directed enzyme evolution via small and effective neutral drift libraries. Nature methods 2008, 5:939-942.

70. Messier N, Roy PH: Integron integrases possess a unique additional domain necessary for activity. Journal of bacteriology 2001, 183:6699-6706.

71. Johansson C, Boukharta L, Eriksson J, Aqvist J, Sundstrom L: Mutagenesis and homology modeling of the Tn21 integron integrase Intl1. Biochemistry 2009, 48:1743-1753.

72. Gravel A, Messier N, Roy PH: Point mutations in the integron integrase Intl1 that affect recombination and/or substrate recognition. Journal of bacteriology 1998, 180:5437-5442.

73. Demarre G, Frumerie C, Gopaul DN, Mazel D: Identification of key structural determinants of the Intl1 integron integrase that influence attC $\times$ attl1 recombination efficiency. Nucleic acids research 2007, 35:6475-6489.

74. Frumerie C, Ducos-Galand M, Gopaul DN, Mazel D: The relaxed requirements of the integron cleavage site allow predictable changes in integron target specificity. Nucleic acids research 2010, 38:559-569.

75. Edgar RC: MUSCLE: multiple sequence alignment with high accuracy and high throughput. Nucleic acids research 2004, 32:1792-1797.

76. Drouin F, Melancon J, Roy PH: The Intl-like tyrosine recombinase of Shewanella oneidensis is active as an integron integrase. Journal of bacteriology 2002, 184:1811-1815.
77. Collis CM, Kim MJ, Partridge SR, Stokes HW, Hall RM: Characterization of the class 3 integron and the site-specific recombination system it determines. Journal of bacteriology 2002, 184:3017-3026.

78. Hansson K, Sundstrom L, Pelletier A, Roy PH: Intl2 integron integrase in Tn7. Journal of bacteriology 2002, 184:1712-1721.

79. Biskri L, Bouvier M, Guerout AM, Boisnard S, Mazel D: Comparative study of class 1 integron and Vibrio cholerae superintegron integrase activities. Journal of bacteriology 2005, 187:1740-1750.

80. Martinez E, de la Cruz F: Genetic elements involved in Tn21 site-specific integration, a novel mechanism for the dissemination of antibiotic resistance genes. The EMBO journal 1990, 9:1275-1281.

81. Erill I, O'Neill MC: A reexamination of information theory-based methods for DNA-binding site identification. BMC bioinformatics 2009, 10:57.

82. Bhargava N, Erill I: xFITOM: a generic GUI tool to search for transcription factor binding sites. Bioinformation 2010, 5:49-50.

83. Schneider TD: Information Content of Individual Genetic Sequences. Journal of Theoretical Biology 1997, 189:427-441.

84. Abella M, Campoy S, Erill I, Rojo F, Barbe J: Cohabitation of two different lexA regulons in Pseudomonas putida. Journal of bacteriology 2007, 189:8855-8862.

85. Cheo DL, Bayles KW, Yasbin RE: Elucidation of regulatory elements that control damage induction and competence induction of the Bacillus subtilis SOS system. Journal of bacteriology 1993, 175:5907-5915.

86. Movahedzadeh F, Colston MJ, Davis EO: Characterization of Mycobacterium tuberculosis LexA: recognition of a Cheo (Bacillus-type SOS) box. Microbiology (Reading, England) 1997, 143(Pt 3):929-936.

87. Fernandez de Henestrosa AR, Rivera E, Tapias A, Barbe J: Identification of the Rhodobacter sphaeroides SOS box. Molecular microbiology 1998, 28:991-1003.

88. Erill I, Campoy S, Mazon G, Barbe J: Dispersal and regulation of an adaptive mutagenesis cassette in the bacteria domain. Nucleic acids research 2006, 34:66-77.

89. Thompson JD, Higgins DG, Gibson TJ: CLUSTAL W: improving the sensitivity of progressive multiple sequence alignment through sequence weighting, position-specific gap penalties and weight matrix choice. Nucleic acids research 1994, 22:4673-4680.

90. Notredame C, Higgins DG, Heringa J: T-Coffee: A novel method for fast and accurate multiple sequence alignment. Journal of molecular biology 2000, 302:205-217

91. Castresana J: Selection of conserved blocks from multiple alignments for their use in phylogenetic analysis. Molecular biology and evolution 2000, 17:540-552.

92. Guindon S, Gascuel O: A simple, fast, and accurate algorithm to estimate large phylogenies by maximum likelihood. Systematic biology 2003, 52:696-704.

93. Page RD: TreeView: an application to display phylogenetic trees on personal computers. Comput Appl Biosci 1996, 12:357-358.

94. Mesquite: a modular system for evolutionary analysis. Version 2.72. [http://mesquiteproject.org].

95. Pagel M: The maximum likelihood approach to reconstructing ancestral character states of discrete characters on phylogenies. Systematic biology 1999, 48:612-622.

96. Schluter D, Price T, Mooers AO, Ludwig D: Likelihood of ancestor states in adaptive radiation. Evolution 1997, 51:1699-1711.

97. Omland KE: The assumptions and challenges of ancestral state reconstructions. Systematic biology 1999, 48:604-611.

98. Datsenko KA, Wanner BL: One-step inactivation of chromosomal genes in Escherichia coli K-12 using PCR products. Proceedings of the National Academy of Sciences of the United States of America 2000, 97:6640-6645.

99. Abella M, Erill I, Jara M, Mazon G, Campoy S, Barbe J: Widespread distribution of a lexA-regulated DNA damage-inducible multiple gene cassette in the Proteobacteria phylum. Molecular microbiology 2004, 54:212-222.

100. Crooks GE, Hon G, Chandonia JM, Brenner SE: WebLogo: a sequence logo generator. Genome research 2004, 14:1188-1190.

doi:10.1186/1759-8753-2-6

Cite this article as: Cambray et al.: Prevalence of SOS-mediated contro of integron integrase expression as an adaptive trait of chromosomal and mobile integrons. Mobile DNA 2011 2:6. 